\title{
Palliative Care Principles and Anesthesiology Clinical Practice: Current Perspectives
}

\author{
Giulia Catalisano (D) \\ Mariachiara Ippolito (1D) \\ Claudia Marino' \\ Antonino Giarratano ${ }^{1,2}$ \\ Andrea Cortegiani ${ }^{1,2}$ \\ 'Department of Surgical, Oncological and \\ Oral Science (Di.Chir.On.S.), University \\ of Palermo, Palermo, Italy; ${ }^{2}$ Department \\ of Anesthesia, Intensive Care and \\ Emergency, Policlinico Paolo Giaccone, \\ Palermo, Italy
}

Background: Palliative care is a person-centered approach aiming to relieve patient's health-related suffering and it is often needed when caring for critically ill patients to manage symptoms and identify goals of care.

Aim: To describe the integration of palliative care principles in anesthesiology clinical practice, within and outside the ICU and to analyze the additional challenges that COVID19 pandemic is posing in this context.

Methods: For the purpose of this review, PubMed database was searched for studies concerning palliative care and end of life care, in contexts involving anesthesiologists and intensivists, published in the last 5 years.

Results: Anesthesiologists and intensivists integrate palliative care within their daily practice providing symptoms management as well as family counseling. High-quality communicational skills are fundamental for anesthesiologists and intensivists especially when interfacing with surrogate decision makers in the ICU or with patients in the preoperative setting while discussing goals of care. Coronavirus disease 2019 (COVID-19) pandemic has challenged many aspects of palliative care delivery: reduced family presence within the ICU, communication with families through phone calls or video calls, patient-physician relationship mediated by bulky personal protective equipment and healthcare workers physical and psychological distress due to the increased workload and limitations in resources are some of the most evident.

Conclusion: Anesthesiologists and intensivists are increasingly facing challenging clinical situations where principles and practice of palliative care have to be applied. In this sense, increasing knowledge on palliative care and providing specific training would allow to deliver high-quality symptom management, family counseling and end of life guidance in critical care settings. COVID-19 pandemic sets additional difficulties to palliative care delivery.

Keywords: palliative care, end of life care, intensive care unit, anesthesiologist, intensivist, COVID-19

\section{Introduction}

According to the World Health Organization, the aim of palliative care is the prevention and relief of health-related suffering of patients facing problems associated with life-threatening illness and their families. Palliative care results in a comprehensive and person-centered approach, addressing physical, psychological, social and spiritual suffering. ${ }^{1}$ Goals of the intensive care are to reduce morbidity and mortality associated with critical illness, support organ function and restore health. ${ }^{2}$

The anesthesiologist job covers a broad spectrum of different tasks depending on countries and hospitals: perioperative risk assessment, intraoperative and
Correspondence: Andrea Cortegiani Department of Surgical, Oncological and Oral Science (Di.Chir.On.S), University of Palermo, Palermo, Italy Tel +390916552730

Email andrea.cortegiani@unipa.it 
postoperative management of patients, pain management of both hospitalized and out-patients, physicians of intensive care units (ICUs) and medical emergency team are the most common. As such, anesthesiologists deal with palliative care in several aspects of their clinical practice. One of the main issues is providing palliative care to critically ill patients admitted to ICU to whom intensive treatments would not be curative. However, palliative care is not only related to the end of life care, and the concept has evolved over time, now focusing on patients' needs more than on prognosis. ${ }^{3,4}$ Indeed, also patients with a full-code status may need palliation of suffering in its broader significance, from symptoms relief to psychological assistance. ${ }^{3,5}$ Similarly, anesthesiologists may resort to palliative care measures also in the emergency setting, for patients whose admission to ICU is not being considered, in the management of pre and post-surgical patients or when treating outpatients for cancer and noncancer related pain. ${ }^{6}$

The ongoing Coronavirus disease 2019 (COVID-19) pandemic is having a considerable impact on healthcare systems organization and on daily clinical practice. Anesthesiologists and intensivists are among those who are most experiencing these changes, and therefore palliative care is expected to further mutate during this time. ${ }^{7}$

In this narrative review, we describe the integration of palliative care principles in anesthesiology clinical practice, within and outside the ICU, in light of evidence from the literature of the last 5 years and analyze the additional challenges that COVID-19 pandemic is posing in this context.

\section{Methods}

\section{Search Strategy}

For the purpose of this review, PubMed database was searched for studies concerning palliative care and end of life care, in context involving anesthesiologists, published from 2016 up to the 29th of March 2021. The search included the following keywords: "anesthesia", "anesthesiologist", "ICU", "intensive care unit", "palliative care" and "end of life care" as exact phrases and as combination of broad subject headings according to database syntax. Three authors (GC, MI and CM) independently screened the retrieved records for relevant articles. The process of inclusion and exclusion is detailed in the PRISMA flow diagram, provided as Figure 1.

\section{ICU and Palliative Care}

\section{Background and Models}

Even though intensive care units (ICUs) see a great variability regarding physicians' area of specialty ranging from internists, pulmonologists, anesthesiologists to surgeons, physicians specialized in anesthesia and intensive care may often have a leadership role in the ICU medical teams, especially in Europe. ${ }^{8-10}$

According to the Lancet Commission report, the alleviation of the burden of pain, suffering, and severe distress associated with life-threatening conditions and with end of life is a global health and equity imperative. ${ }^{11}$ Still, a high prevalence of unmet palliative care needs is registered in countries such as the United States (US), especially in acute care settings. ${ }^{12}$ Since the ICU setting is burdened by high mortality and high suffering, providing palliative care to critically ill patients and their families is a major goal of ICU care. Teno et al in a retrospective cohort study among Medicare decedents, observed that in 2015 up to the $29 \%$ of decedents accessed an ICU during the last 30 days of life. ${ }^{13}$ Moreover, many ICU patients present unrelieved and distressing symptoms that could be addressed through early palliative care assessment. ${ }^{5,14}$

A prospective, observational study investigating the symptoms experienced by 171 ICU patients at high risk of death over a 14-day period, concluded that in more than half of the 405 symptom assessments performed, patients reported the presence of tiredness $(74.7 \%)$, thirst $(70.8 \%)$ and anxiety $(57.9 \%) .{ }^{15}$ Data suggest that integration of palliative care in the ICU improves quality of life, caregiver burden and might lead to decreased hospital and ICU length of stay, although this last point is controversial. ${ }^{16-19}$

There are two main models to provide palliative care in ICU. ${ }^{5,16}$ The consultative model is based on the fixed presence of palliative care specialist physicians consulted by intensivists upon patients meeting triggers for initiation of palliative care. ${ }^{5,20}$ Even though the number of palliative care specialists is increasing, their availability is not sufficient to cover the raising need for palliative care in the ICU population. ${ }^{4,5}$ The lack of specialized palliative care teams within healthcare structures might hinder the integration of palliative care approach for ICU patients. ${ }^{21}$

The integrative model seeks to address the existing need for palliative care interventions within daily practice of ICU clinicians for all patients and families facing critical illness. ${ }^{5}$ As such, intensivists and the whole ICU team take responsibility for primary palliative care needs of 


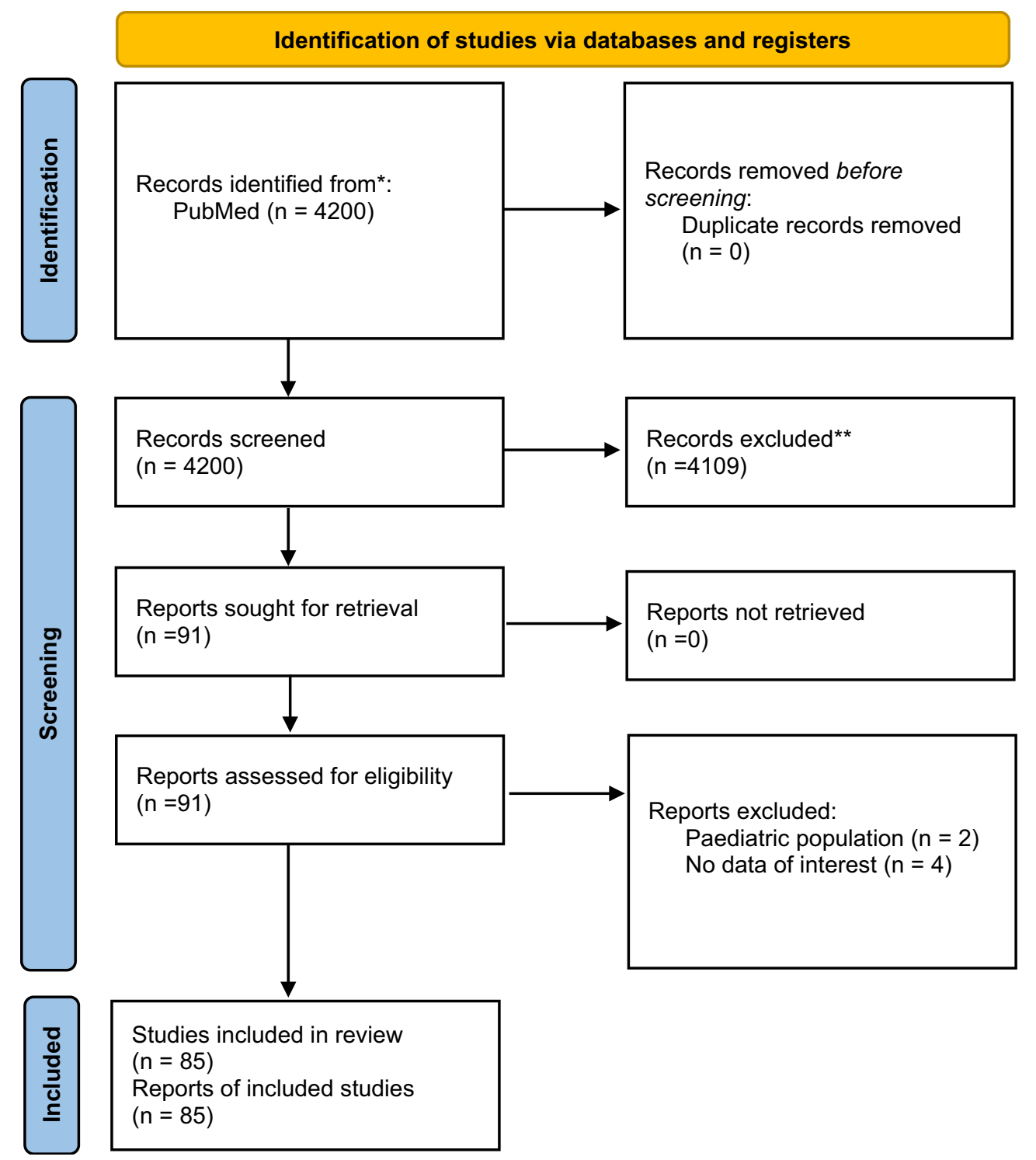

Figure I PRISMA 2020 flow diagram for new systematic reviews which included searches of databases and registers only.

Note:Adapted from Page MJ, McKenzie JE, Bossuyt PM, Boutron I, Hoffmann TC, Mulrow CD, et al. The PRISMA 2020 statement: an updated guideline for reporting systematic reviews. BMJ 2021;372:n7I. doi: 10.1136/bmj.n71. ${ }^{92}$

patients and address the complex setting of end of life, also interfacing with family and surrogate decision makers. $^{3}$

Ideally, a mixed model would probably benefit patients' care the most, having intensivist managing daily primary palliative care needs and consulting palliative care specialists upon most difficult cases. ${ }^{4,22}$ Moreover, palliative care consultants are more prone to offer spiritual support and manage documented symptoms, instead of performing symptoms assessment themselves. This aspect highlights the specific competencies of consultative palliative care which, in order to ensure high quality comprehensive care, need complementation by ICU clinicians. ${ }^{20}$

Either way ICU clinicians need knowledge, skills and systems to support their essential role in palliative care. ${ }^{3}$ ICU physicians' training, knowledge, self-confidence and attitudes towards palliative care and end of life care have been surveyed in several countries. ${ }^{23-26}$ A descriptive, cross-sectional and correlational study was conducted through a web based voluntary survey answered by 101 physicians working in Colombia's ICUs. The study reported that over $60 \%$ of the participants did not have the possibility to work with a palliative care specialist in 
the ICU and that the hours of ICU physician's training in palliative care influenced positively palliative care perception and negatively barriers perception, which resulted in an increased interaction with palliative care specialists, especially in relation to emotional and family issues. ${ }^{26}$ Likewise, 805 anesthesiologists working in Italian ICUs responded to a web-based survey endorsed by the Italian society of anesthesia, analgesia, resuscitation and intensive care (SIAARTI) ${ }^{23}$ The study found that in about $70 \%$ of the cases there was no palliative/supportive care team in the hospital. Even though systematic symptoms' recording is pivotal in palliative care, the same survey noted a general lack of it, with up to $20 \%$ of the respondents affirming that none of the symptoms among pain, dyspnea, thirst, nausea, anxiety and depression was systematically recorded. Interestingly, in the same study $84 \%$ of respondents stated they did not receive a specific training in palliative/supportive care. ${ }^{23}$ In line with this aspect are also a survey-based study held in India to which 202 ICU physicians responded of which $66.8 \%$ were anesthesiologists ${ }^{24}$ and a multicenter prospective observational study on 192 ICU physicians $(71.8 \%$ were anesthesiologists) from ten different German hospitals which found that physicians' self-confidence was not necessarily related to their level of knowledge specifically in palliative care, while the few physicians having an additional certificate in either pain $(6.6 \%)$ or palliative medicine $(5.8 \%)$ had a positive correlation both with self-confidence and knowledge. $^{25}$

\section{Palliative Care Integration for Critically III Patients}

The conception that palliative care is purely associated with end of life may lead to reticence for early referral. ${ }^{17}$ In a retrospective cohort study including 78 hospitals in Pennsylvania, Ashana et al found that the availability of ICU end of life resources was not associated with increased in-hospital mortality, mortality up to 90 days after hospital admission or increased resources utilization at end of life. On the other hand, they found an increased likelihood of discharge to hospice $(\mathrm{OR}=1.58 ; 95 \% \mathrm{CI}=$ 1.1 to 2.24$).{ }^{27}$ According to a systematic review about the impact of palliative care consultation in the ICU on length of stay the patients with a palliative care consultation, when compared to those who did not, showed a trend toward reduced length of stay, while mortality was similar in both groups. ${ }^{19}$ These findings may encourage ICU clinicians, surrogates and patients to start palliative care interventions to alleviate pain and other symptoms without hastening end of life. ${ }^{17}$ Therefore, palliative care should be part of the standard of care of all critically ill patients.

\section{ICU Family-Centered Care}

Palliative care should be patient tailored and family centered. Family involvement has a pivotal role in patients care and recovery. Healthcare teams should provide support for families of seriously ill patients. According to the recommendations published on 2017 guidelines for family-centered care in ICU, relatives of critically ill patients should be offered open flexible family presence at the bedside (Figure 2). ${ }^{28}$ Open doors ICU models require the transformation of family members from ICU visitors to partners in care, if they wish so. ${ }^{29}$ It is also recommended to offer family members of critically ill patients the option of participating to interdisciplinary team rounds. ${ }^{28}$ According to a multicenter observational study on family participation to ICU rounds in 7 Canadian ICUs, family attendance during ICU rounds fosters relationship building, facilitates shared decision-making and enhances communication. Moreover, it was found that the duration of rounds would only modestly increase, with no impact on trainee teaching and willingness to discuss sensitive information. ${ }^{30}$ The positive role of family presence in supporting the patient's care and recovery is underlined by a before and after study comparing an extended visitation model (12 hours per day) with a restrictive visitation model ( 4.5 hours per day) in 286 patients of a medical-surgical ICU. In this study, the extended visitation model was associated with a reduction in delirium occurrence and its length. ${ }^{31}$ Delirium prevention is particularly relevant, especially in the elderly patients, who are most vulnerable to functional decline and cognitive impairment. ${ }^{32}$ Family presence and protocolized family support interventions for enhanced communication also influence ICU length of stay, reducing it. $^{31,33}$

Still, ICU mortality and 6 months mortality after admission remains high, especially in elder population often burdened by low long-term recovery of functional status. $^{34,35}$ A relevant number of patients discharged from the ICU may experience the post intensive care syndrome (PICS) with physical, cognition and mental impairments, affecting the quality of life of patients and families. ${ }^{29}$ Moreover, according to a prospective cohort study on 125 patients discharged from the ICU after mechanical 


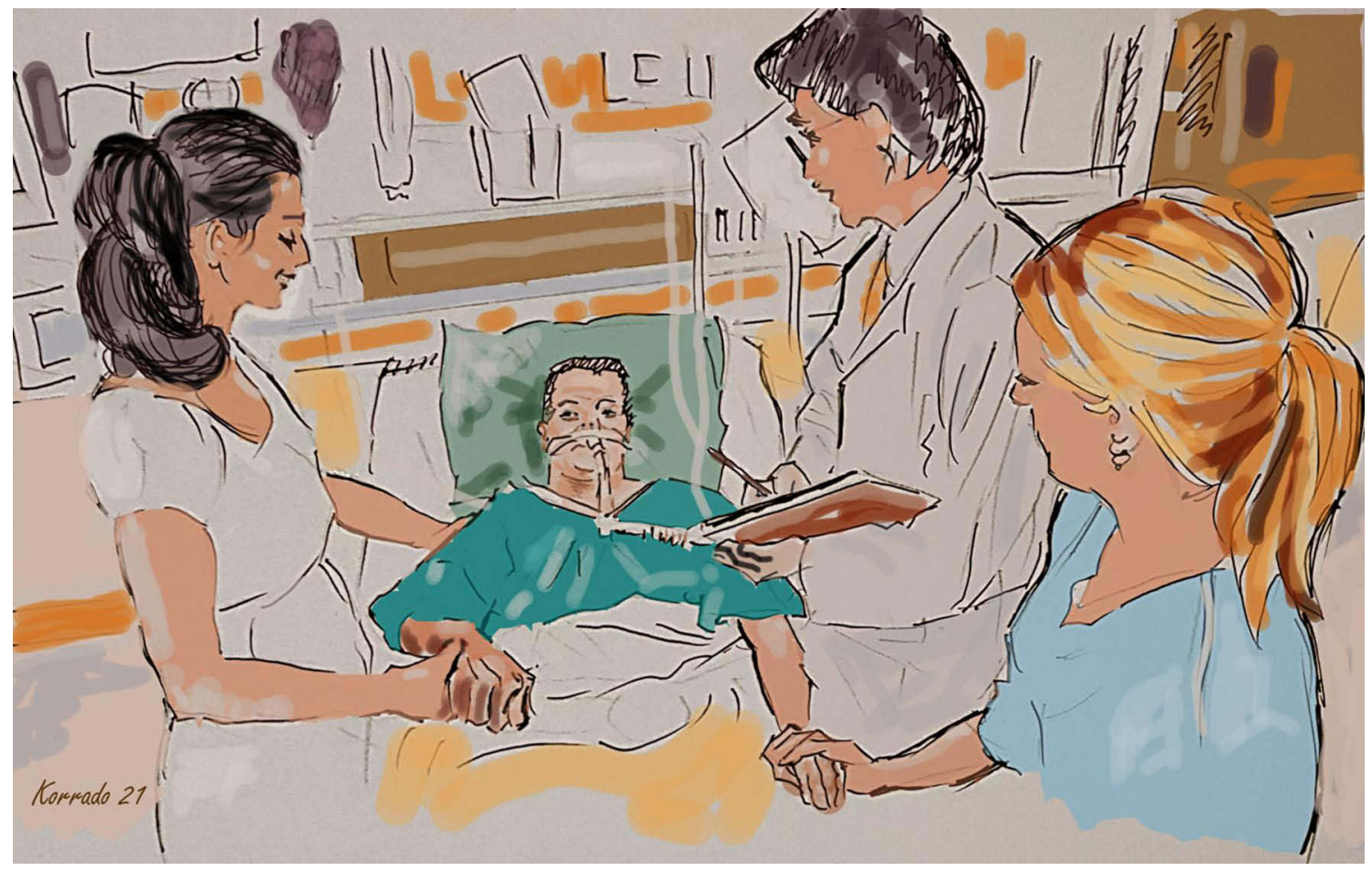

Figure 2 The illustration shows an example of interaction and cooperation between family members and clinicians at the patient's bedside.

ventilation aged 65 or more, older ICU survivors have a high burden of palliative care needs that persist at 1 month after discharge, fatigue being the most prevalent symptom. ${ }^{36}$ Family engagement is one of the many important interventions that ICU staff can apply in order to prevent PICS. ${ }^{29,37}$ Lastly, it is also important to consider that family members themselves may also experience psychological and physical risks when caring for critically ill patients. ${ }^{38,39}$ The creation of opportunities for closeness and family care rituals for relatives involved with providing care for a loved one in the ICU was associated with a reduced occurrence of symptoms of post-traumatic stress disorder (PTSD) in family members at 90 days after patient's death or ICU discharge. ${ }^{40}$

\section{End of Life Decision Making}

The assessment of patient's condition with the aim of looking for palliative care needs and assessing patientcentered goals of care is a core part of the intensive care medicine. When the full-code status may not benefit the patient anymore, the process of care may converge towards end of life care and withholding or withdrawal of non-beneficial treatments. ${ }^{41}$ Ethicus-2, a prospective observational study of 22 European ICUs previously included in the Ethicus-1 (1999-2000) study, collected data about patients who died or had any limitation of lifeprolonging therapies in the 2015-2016 comparing it to the 1999-2000 cohort. Limitations in life-prolonging therapies occurred $21.4 \%$ times more frequently (1601 patients [89.7\%] in 2015-16 vs 1918 [68.3\%] in 1999-2000; difference, $21.4 \%$ [ $95 \%$ CI, $19.2 \%$ to $23.6 \%$ ]; $p<0.001$ ) in the recent years, suggesting an increasing adherence to end of life practices in European ICUs. ${ }^{42}$ Other studies reported that early triggered palliative care consultation was associated with transition in code status (DNR/DNI) and with hospice referral. ${ }^{18,43}$ There is considerable worldwide variability in decisions to withhold/withdraw lifesustaining treatments ${ }^{44}$ which might be influenced by cultural, religious and social factors as well as by the ICUs ethical climate. ${ }^{16,45}$ Even so, an intensivist should avoid disproportionate and non-beneficial treatments. Decisions surrounding end of life care should be made by the entire ICU team of clinicians, and not by a subset of members. ${ }^{14}$ Consensus among the entire clinical team is one of the first and most important steps in withholding/withdrawal of non-beneficial treatments for ICU clinicians, who are at higher risk of moral distress and burnout. ${ }^{41,46-48}$

Patients admitted to ICU are usually not able to express their preferences about end of life care directly. ${ }^{16}$ Therefore, intensivists have to acknowledge any advance 
directive and interact with patient's surrogate decision maker and family. During patient's stay in ICU, physicians are recommended to use routine interdisciplinary family conferences to receive directives, discuss goals, assess family needs, cultivate mutual trust and reduce conflicts between the treating clinicians and the family members. ${ }^{28}$ The quality of these conferences is even more important when end of life care becomes the main topic to discuss. Surrogate decision makers often are not prepared for this role, and struggle with decisions related to goals of care, experiencing higher levels of distress compared to the other family members. ${ }^{49,50}$ A secondary analysis of a prospective, multicenter cohort study of audiorecorded clinician-family conferences enrolling 249 patients and involving 450 surrogates and 141 clinicians found that a quarter of all clinician-family conferences about prognosis and goals of care for critically ill patients lacked important elements of communication about patient's values and preferences, of note only $5.4 \%$ of the clinicians involved were anesthesiologist and $2.7 \%$ had internal medicine and anesthesiology as specialty. ${ }^{51}$ Interestingly, a prospective multicenter cohort study in 12 ICUs located in 5 different states of the US found that $45 \%$ of the 245 surrogate decision makers enrolled had physiciansurrogate discordance about patient's prognosis. The discordance was associated both with misunderstandings of physicians' assessment of prognosis (48\% of cases) and with surrogates holding more optimistic beliefs compared with what they perceived to be the physician's assessment of prognosis. Interestingly, in this study the $7.3 \%$ of the physicians were anesthesiologists. This study underlines the need for improvement of communicational skills surrounding prognosis discussion and the need to attend to the emotional and psychological factors that influence surrogates' prognostic expectations. ${ }^{52}$ Low quality communication may be a contributing cause for surrogateclinician disagreement about appropriateness of treatment. According to Wilson et al, who conducted a prospective, observational study in 6 adult ICUs in the US and Hungary with 151 patients enrolled, surrogate-clinician disagreement may arise in one-third of ICU patients and was associated with prognostic discordance, lower satisfaction and trust in the ICU team. ${ }^{53}$ Being aware of factors that may impair communication and influence end of life decision making can facilitate interventions aiming towards high quality, compassionate and culturally sensitive decision making. ${ }^{54}$ For example, a retrospective cohort study of 779 ICU patients with limited English proficiency found that these patients have significantly lower rates of do not resuscitate (DNR) orders, advance directive completion and palliative care before death. Decision to limit lifesupport treatments also takes 3.8 days longer than in general population. ${ }^{55}$ Clinicians interviewed on this topic identified elements of additional difficulty such as less frequent and modified communication, impaired ability to assess patients' and families' understanding of serious topics and impaired patient/clinician relationship building. ${ }^{54}$

In light of this, high quality communication and attention about surrogate's psychological wellbeing should be enhanced. A pre-post intervention study on 80 primary family members interviewed between the third and the fifth day of admission of the patient to the ICU, compared structured communication with usual communication between ICU staff and family. Families enrolled in the structured communication group reported higher satisfaction regarding ease of obtaining the information, and the consistency of the information provided. Moreover, ICU staff and family expectations about hospital survival were better correlated in the intervention group. ${ }^{56}$ White et al structured a cluster-randomized trial involving patients with a high risk of death and their surrogates in five ICUs, in order to compare a family-support intervention delivered by the interprofessional ICU team with the standard care. The study showed no significant difference between the two groups regarding surrogates' burden of psychological symptoms at 6 months. Conversely, surrogates rating of communication and the centeredness of patient and family were better rated in the intervention group. Additionally, the intervention group registered a shorter ICU length of stay. ${ }^{50}$

An evaluation of the overall experience of family members of patients who died in the ICU may give useful feedback to ICU clinicians as to which area of their work should be improved and help them identify families who are at higher risk for difficult bereavement. CEASAR 15item questionnaire investigates several core aspects of ICU care, from palliative care needs to communication and general satisfaction rating. The CEASAR score 21 days after the patient's death is strongly correlated with the presence during the following months of symptoms of anxiety, depression, PTSD and complicated grief. $^{57}$ Knowing how well we take care of our patients and their families is the basis for future interventions apt to 
ameliorate the difficult experience of having a loved one in the ICU and to diminish relatives burden after their loss.

\section{Palliative Care Outside the ICU}

Anesthesiologists encounter patients that would benefit from receiving palliative care also outside the ICU. Palliative care team consultations, including goal of care conversations, may help avoiding disproportionate life sustaining treatments. ${ }^{58}$ Since not all hospitals provide such services, calls about critically ill patients with poor prognosis are often directed to the medical emergency team (MET). METs can be staffed by physicians with various specialties (anesthesiologists, internists or doctors without a specific specialty) and nurses. In several cases the MET has to initiate discussions surrounding treatment limitations, therefore needing training in palliative care in order to recognize patients who would benefit from it and are not suited for ICU admission. ${ }^{59}$ Screening and referral for palliative care when consulting on wards or ED patients is feasible and palliative care interventions should be implemented especially when transfer of terminally ill patients to hospice care cannot be done for practical reasons. ${ }^{60,61}$

In preoperative settings, the anesthesiologist can recognize triggers for primary palliative care interventions such as optimization of symptoms management or goals of care discussions. This is important when dealing with frail patients who are going to undertake surgery and are at increased risk for major post-operative morbidity and shortand long-term mortality. Anesthesiologists should therefore carefully assess patients aged over 60 for frailty. In this type of patient, discussing goals of care in relation to the intra and post-operative time (code status, preferences about postsurgery level of care and life-prolonging interventions) may be of relevance. ${ }^{62}$ Pursuing enhanced recovery after surgery, there are ways for the anesthesiologist to guide postoperative care including multimodal pain management, delirium prevention, optimization of complicated patients after surgery and nausea management. ${ }^{6}$ Regional anesthesia and analgesia techniques may play a role in this scenario as well as in treatment and management of cancer-related pain. ${ }^{6,63}$ All in all, the anesthesiologist's skill set could make him/her an engaged partner in palliative care also in the context of hospice care. $^{64}$

\section{Palliative Care and COVID-I 9}

Coronavirus diseases 2019 (COVID-19) pandemic posed an additional challenge to palliative care, due to the surge in the number of critical care patients. ${ }^{7}$ Moreover, the increased workload, the high mortality and the general pandemic setting saw healthcare workers report symptoms of psychological and physical distress. ${ }^{65-67}$

\section{Inside the ICU}

Since the beginning of COVID-19 pandemic many ICUs have witnessed a transition from the much-advocated family centered and open doors ICU model to the almost complete closure of ICUs to non-clinical staff. The limitations put into place to prevent the spread of SARS-CoV-2 changed the clinical practice in all healthcare facilities and especially in ICUs. ${ }^{28,68}$

Families were prevented from visiting their hospitalized relatives because of the high risk of infection and, especially at the beginning of the pandemic, also due to personal protective equipment (PPE) shortage. $^{69}$ Limitations in family access to ICUs are still common even though some institutions are allowing visits to some extent, especially when dealing with end-of-life situations. $^{70,71}$ This aspect appears to be burdensome for all the people participating to ICU care: patients may experience depression and anxiety; relatives do not have the chance to physically interact with their loved ones and personally observe and assist in treatment and caring process. The effect of these limitations may increase the development of anxiety, post-traumatic stress and complicated grief for families (Figure 3). ${ }^{68,72,73}$ The constant wearing of PPE hindered the possibility of clinicians to communicate in a satisfactory and empathic way with patients. $^{74}$ Semi-structured interviews of 27 members of ICU staff report that clinicians, being the only ones allowed person to person contact with patients, felt a strong responsibility to compensate for the absence of relatives. This scenery worsened even more towards end of life when the absence of rituals was perceived as potentially harmful for families. ${ }^{75}$

Intensivists had to resort to other means of communication given the scarcity of opportunity for face-to-face meetings with relatives. Phone calls and video calls were used to keep contact with families, provide updates on the health state of patients and to better understand their values, wishes or advanced directives for end-of-life care. $^{74}$ Telephone communication precluded non-verbal clues increasing the difficulty to deal with families' emotions. ${ }^{75}$ Also communicating with patients' relatives through phone calls might elicit a lower number of conversations concerning goals of care. ${ }^{75,76}$ Moreover, in hospitals with the most overwhelmed conditions, the possibility to hold such communications routinely may have 


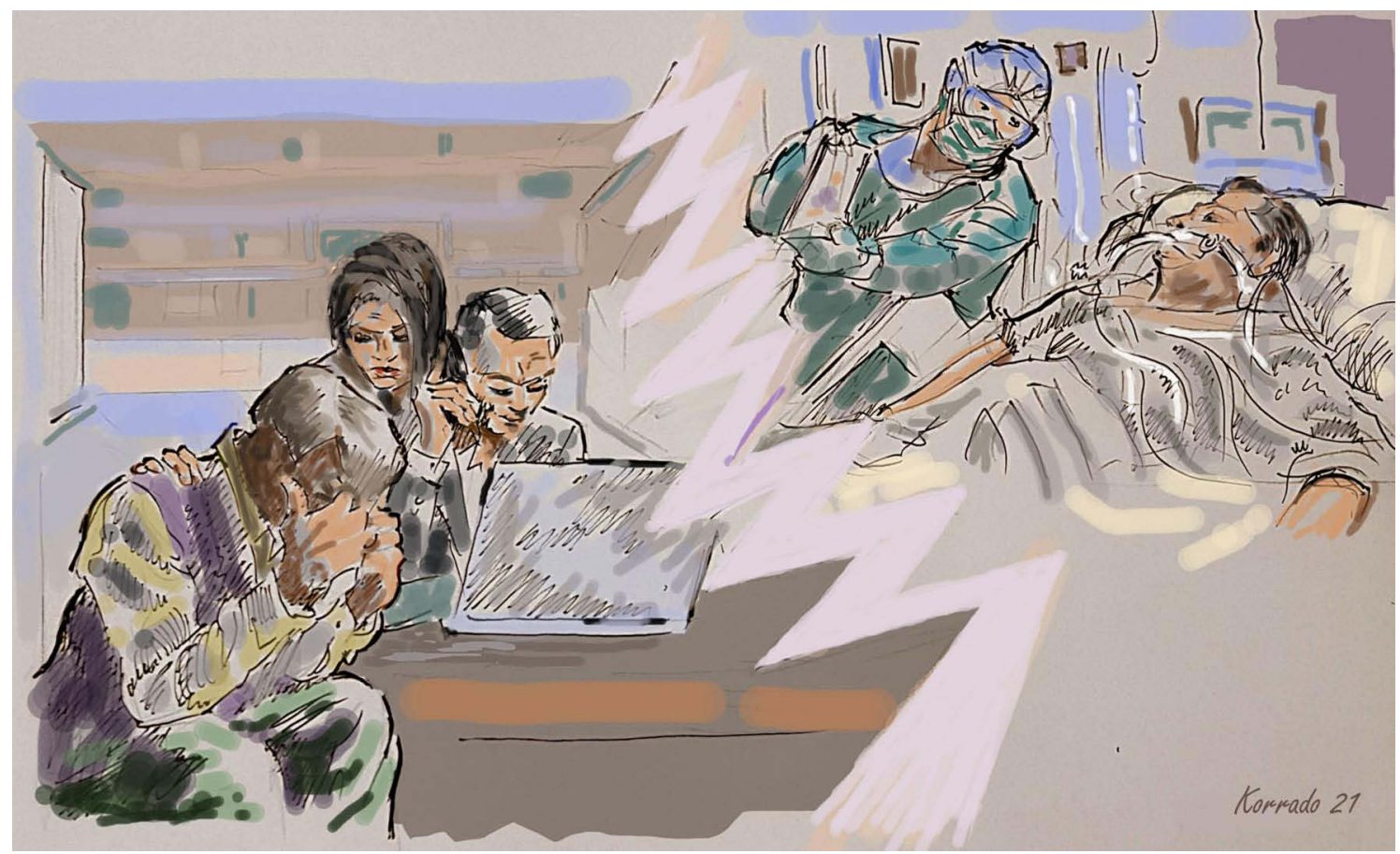

Figure 3 The illustration shows how communication between patient, family and clinicians takes place during COVID-19 pandemic, using digital devices (tablet and laptop).

been scarce or null. Clarifying patient's code status among the treating professionals also is particularly important, especially during COVID-19 pandemic, to ensure all the members of the treating team have a clear idea of the goal of care for each patient. For example, disproportioned cardiopulmonary resuscitation (CPR) could be harmful both for families, who are at risk of psychological distress and may rely on unachievable goals or improvements, but also for healthcare workers performing advanced life support maneuvers with augmented risk of aerosolization and strain of PPE resources. ${ }^{77}$ Additionally, there is a large debate on the outcomes of COVID-19 patients undergoing CPR. ${ }^{78}$ Consultation of palliative care teams, when available, helped clarifying advance directives and minimize futile resuscitation efforts. ${ }^{79,80}$ Some institutions registered an increase in palliative care consultations during COVID19 outbreak $^{81}$ even though it is reported that palliative care consultations often happened late during hospitalization and in a minority of patients. ${ }^{76,82}$ When a specialty palliative care team was not available strategies of integrative primary palliative care delivery were adopted by anesthesiologists trying to optimize patient's comfort while minimizing staff exposure to infection. ${ }^{7,83}$

COVID-19 patients are at greater risk of developing delirium due to the possible direct effect of SARS-CoV-2 on central nervous system, the induction of inflammatory mediators and iatrogenic factors such as prolonged mechanical ventilation, the use of sedation and muscular blocking agents to minimize asynchronies during prone positioning and hindered communication with both family members and healthcare professionals wearing PPE. Intervention on environmental factors like reorientation, family video calls, in-bed mobility and psychological support may prevent and help manage delirium and may also lead to a shorter ICU length of stay. ${ }^{84}$

Lastly, in order to guarantee high-quality palliative care in the context of COVID-19 pandemic a multistep strategy has been proposed which adjusts palliative care services to hospitals' capacity level (conventional, contingency and crisis capacity). ${ }^{85}$

\section{Outside the ICU}

The prolonged lockdowns that many countries adopted to contain the spread of SARS-CoV-2, the decreased capacity of healthcare structures and the strong fear of already fragile patients to get infected changed the practice of palliative care and pain management. Palliative care physicians initially saw a decrease in the number of outpatients' access to pain and palliative care clinics, with foreseeable effects to cancer and non-cancer pain management such as withdrawal and reappearance of symptoms. 
The use of telemedicine was suggested as an alternative to clinic appointments in selected cases. ${ }^{86-88}$

In the pandemic context physicians are advised to engage in discussions about goals of care and advance care planning with patients suffering from severe chronic diseases who are among the high risk population for hospitalization or ICU admission in case of severe acute illness. ${ }^{7,77}$ The presence of advance directives may help the clinician during triage for ICU admission in order to best allocate resources and provide proper cures for each patient. One of the issues at the beginning of the pandemic was the possibility of reaching full hospital capacity, with consequent lack of ICU beds and ventilators. Even before the pandemic breakout it was shown that ICU occupancy was negatively associated with the odds of ICU admission ${ }^{89}$ so, due to possible resource-limited circumstances, clinicians were given ethical and practical recommendations to face this scenario especially during triage for ICU admission. ${ }^{71,90,91}$ On the other hand, other physicians report that in spite of the increased number of critical patients the triage admission criteria to ICU had not changed. $^{75}$

\section{Strengths and Limitations}

Our review gives an overview of the several contexts where an anesthesiologist may confront his/her work with the necessity of palliative care interventions. It also stresses the need for anesthesiologists to implement clinical knowledge in the field as well as good communicational skills to use with patients and families, especially when dealing with end-of-life situations. There is a huge variability in the composition of ICUs' physician staff and medical emergency teams, therefore studies held in different countries may include a different proportion of anesthesiologists within their sample. Moreover, studies including physicians with different specialties do not always give specific data for each specialty. Investigating further physicians' approach to palliative care in ICU depending on their specialty might be of interest for future studies.

Regarding limitations, although we perform a systematic review of the literature, the nature and structure of this review is narrative. Thus, no formal quality assessment or quantitative synthesis of available evidence were performed. Moreover, we did not specifically discuss the pivotal role of other medical specialties (eg surgeons) or other healthcare workers in the care of critically ill patients and their relatives (eg critical care nurses, psychologists) in the context of our review topic.

\section{Conclusion}

Anesthesiologists and intensivists are increasingly facing challenging clinical situations where principles and practice of palliative care have to be applied. In this sense, providing specific training to increase knowledge on palliative care would allow to deliver high-quality symptom management, family counseling and end of life guidance in critical care settings. COVID-19 pandemic is setting additional difficulties on several levels. Interaction between patients, clinicians and families is hindered by the limited opportunities for face-to-face meetings with physicians and family visiting chances, with possible ethical questions arising especially when faced with hospital full capacity. Increasing knowledge on palliative care and providing specific training to physicians would allow to deliver high-quality symptom management, family counseling and end of life guidance in critical care setting.

\section{Abbreviations}

COVID-19, Coronavirus disease 2019; CPR, cardiopulmonary resuscitation; DNI, do not intubate; DNR, do not resuscitate; ED, emergency department; ICU, intensive care unit; MET, medical emergency team; PICS, post intensive care syndrome; PPE, personal protective equipment; PTSD, post-traumatic stress disorder; US, United States.

\section{Acknowledgments}

We thank Dr. Antonio Corrado ("Korrado"), experienced anesthesiologist, for providing the figures.

\section{Author Contributions}

GC conceived the content, drafted the manuscript, approved the final version to be submitted. MI, CM, AG helped in writing the manuscript and revised it critically for important intellectual content, approved the final version to be submitted. AC conceived the content, drafted the manuscript, approved the final version to be submitted. All authors have agreed on the journal to which the article will be submitted, gave final approval of the version to be published, and agree to be accountable for all aspects of the work. 


\section{Disclosure}

All authors report no competing interests in this work.

\section{References}

1. Palliative care. Available from: https://www.who.int/health-topics/pal liative-care. Accessed September 16, 2021.

2. Cook D, Rocker G. Dying with dignity in the intensive care unit. N Engl $J$ Med. 2014;370(26):2506-2514. doi:10.1056/nejmra1208795

3. Mathews KS, Nelson JE. Palliative care in the ICU of 2050: past is prologue. Intensive Care Med. 2017;43(12):1850-1852. doi:10.1007/ s00134-017-4828-7

4. Edwards JD, Voigt LP, Nelson JE. Ten key points about ICU palliative care. Intensive Care Med. 2017;43(1):83-85. doi:10.1007/ s00134-016-4481-6

5. Mercadante S, Gregoretti C, Cortegiani A. Palliative care in intensive care units: why, where, what, who, when, how. BMC Anesthesiol. 2018;18(1):1-6. doi:10.1186/S12871-018-0574-9

6. Cobert J, Hauck J, Flanagan E, Knudsen N, Galanos A. Anesthesiaguided palliative care in the perioperative surgical home model. Anesth Analg. 2018;127(1):284-288. doi:10.1213/ANE.0000000000002775

7. Sullivan DR, Curtis JR. A view from the frontline: palliative and ethical considerations of the COVID-19 pandemic. J Palliat Med. 2021;24(2):293-295. doi:10.1089/jpm.2020.0426

8. Hanson CW, Durbin CG, Maccioli GA, et al. The anesthesiologist in critical care medicine: past, present, and future. Anesthesiology. 2001;95(3):781-788. doi:10.1097/00000542-200109000-00034

9. Evans T, Elliott M, Ranieri M, et al. Pulmonary medicine and (adult) critical care medicine in Europe. Eur Respir J. 2002;19 (6):1202-1206. doi:10.1183/09031936.02.00307502

10. ESAIC. Newsletter October 2020: anaesthesia versus intensive care medicine: a deleterious debate. Available from: https://www.esaic. org/esa-news/newsletter-october-2020-anaesthesia-versus-intensivecare-medicine-a-deleterious-debate/. Accessed September 5, 2021.

11. Knaul FM, Farmer PE, Krakauer EL, et al. Alleviating the access abyss in palliative care and pain relief - an imperative of universal health coverage: the Lancet Commission report. Lancet. 2018;391 (10128):1391-1454. doi:10.1016/S0140-6736(17)32513-8

12. Chuang E, Hope AA, Allyn K, Szalkiewicz E, Gary B, Gong MN. Gaps in provision of primary and specialty palliative care in the acute care setting by race and ethnicity. J Pain Symptom Manage. 2017;54 (5):645-653.e1. doi:10.1016/j.jpainsymman.2017.05.001

13. Teno JM, Gozalo P, Trivedi AN, et al. Site of death, place of care, and health care transitions among US medicare beneficiaries, 20002015. JAMA. 2018;320(3):264-271. doi:10.1001/jama.2018.8981

14. Ely EW, Azoulay E, Sprung CL. Eight things we would never do regarding end-of-life care in the ICU. Intensive Care Med. 2019;45 (8):1116-1118. doi:10.1007/s00134-019-05562-9

15. Puntillo KA, Arai S, Cohen NH, et al. Symptoms experienced by intensive care unit patients at high risk of dying. Crit Care Med. 2010;38(11):2155-2160. doi:10.1097/CCM.0b013e3181f267ee

16. Metaxa V. End-of-life issues in intensive care units. Semin Respir Crit Care Med. 2021;42(1):160-168. doi:10.1055/s-0040-1710370

17. Cooksley T. The disappearing dichotomy between critical care and palliative care: integration will enhance patient outcomes. Crit Care Med. 2019;47(11):1667-1668. doi:10.1097/CCM.0000000000 003994

18. Zalenski RJ, Jones SS, Courage C, et al. Impact of palliative care screening and consultation in the ICU: a multihospital quality improvement project. J Pain Symptom Manage. 2017;53(1):5-12. e3. doi:10.1016/j.jpainsymman.2016.08.003

19. Kyeremanteng K, Gagnon LP, Thavorn K, Heyland D, D’Egidio G. The impact of palliative care consultation in the ICU on length of stay: a systematic review and cost evaluation. J Intensive Care Med. 2018;33(6):346-353. doi:10.1177/0885066616664329
20. Wysham NG, Hochman MJ, Wolf SP, Cox CE, Kamal AH. Performance of consultative palliative care model in achieving quality metrics in the ICU. J Pain Sympt Manage. 2016;52:873-877. Elsevier Inc.. doi:10.1016/j.jpainsymman.2016.05.026

21. Hamdan Alshehri H, Olausson S, Öhlén J, Wolf A. Factors influencing the integration of a palliative approach in intensive care units: a systematic mixed-methods review. BMC Palliative Care. 2020;19 (1):1-8. doi:10.1186/s12904-020-00616-y

22. Wysham NG, Hua M, Hough CL, et al. Improving ICU-based palliative care delivery. Crit Care Med. 2017;45(4):e372-e378. doi:10.1097/CCM.0000000000002099

23. Cortegiani A, Russotto V, Raineri SM, Gregoretti C, Giarratano A, Mercadante S. Attitudes towards end-of-life issues in intensive care unit among Italian anesthesiologists: a nation-wide survey. Support Care Cancer. 2018;26(6):1773-1780. doi:10.1007/s00520-017-4014-z

24. Agrawal K, Garg R, Bhatnagar S. Knowledge and awareness of end-of-life care among doctors working in intensive care units at a tertiary care center: a questionnaire-based study. Indian J Crit Care Med. 2019;23(12):568-573. doi:10.5005/jp-journals-1007123293

25. Krautheim V, Schmitz A, Benze G, et al. Self-confidence and knowledge of German ICU physicians in palliative care - a multicentre prospective study. BMC Palliat Care. 2017;16(1):1-8. doi:10.1186/ s12904-017-0244-6

26. Calle MC, Pareja SL, Villa MM, et al. Interactions between intensive care and palliative care are influenced by training, professionals' perceptions and institutional barriers. $J$ Palliat Care. 2020;082585972095136. doi:10.1177/0825859720951361.

27. Ashana DC, Umscheid CA, Stephens-Shields AJ, et al. Determining the association between end-of-life care resources and patient outcomes in Pennsylvania ICUs*. Crit Care Med. 2019;47 (11):1591-1598. doi:10.1097/CCM.0000000000003969

28. Davidson JE, Aslakson RA, Long AC, et al. Guidelines for family-centered care in the neonatal, pediatric, and adult ICU. Crit Care Med. 2017;45(1):103-128. doi:10.1097/CCM.00000000 00002169

29. Vaeza NN, Martin Delgado MC, La Calle GH. Humanizing intensive care: toward a human-centered care ICU model. Crit Care Med. 2020;48(3):385-390. doi:10.1097/CCM.0000000000004191

30. Au SS, Roze Des Ordons AL, Leigh JP, et al. A multicenter observational study of family participation in ICU rounds. Crit Care Med. 2018;46(8):1255-1262. doi:10.1097/CCM.0000000000003193

31. Rosa RG, Tonietto TF, Da Silva DB, et al. Effectiveness and safety of an extended ICU visitation model for delirium prevention: a before and after study. Crit Care Med. 2017;45(10):1660-1667. doi:10.1097/CCM.0000000000002588

32. Tardini F, Pinciroli R, Berra L. The intensive care unit: how to make this unfriendly environment geriatric-friendly. Eur J Surg Oncol. 2020;46(3):379-382. doi:10.1016/j.ejso.2019.12.022

33. Lee HW, Park Y, Jang EJ, Lee YJ. Intensive care unit length of stay is reduced by protocolized family support intervention: a systematic review and meta-analysis. Intensive Care Med. 2019;45 (8):1072-1081. doi:10.1007/s00134-019-05681-3

34. Hua MS, Ma X, Li G, Wunsch H. Derivation of data-driven triggers for palliative care consultation in critically ill patients. $J$ Crit Care. 2018;46:79-83. doi:10.1016/j.jcrc.2018.04.014

35. Leblanc G, Boumendil A, Guidet B. Ten things to know about critically ill elderly patients. Intensive Care Med. 2017;43 (2):217-219. doi:10.1007/s00134-016-4477-2

36. Pollack LR, Goldstein NE, Gonzalez WC, et al. The frailty phenotype and palliative care needs of older survivors of critical illness. $J$ Am Geriatr Soc. 2017;65(6):1168-1175. doi:10.1111/jgs.14799

37. Colbenson GA, Johnson A, Wilson ME. Post-intensive care syndrome: impact, prevention, and management. Breathe. 2019;15 (2):98-101. doi:10.1183/20734735.0013-2019 
38. Sanfilippo F, Ippolito M, Santonocito C, et al. Long-term functional and psychological recovery in a population of acute respiratory distress syndrome patients treated with VV-ECMO and in their caregivers. Minerva Anestesiol. 2019;85(9):971-980. doi:10.23736/ S0375-9393.19.13095-7

39. Cameron JI, Chu LM, Matte A, et al. One-year outcomes in caregivers of critically ill patients. $N$ Engl J Med. 2016;374 (19):1831-1841. doi:10.1056/nejmoa 1511160

40. Amass TH, Villa G, OMahony S, et al. Family care rituals in the ICU to reduce symptoms of post-traumatic stress disorder in family membersa multicenter, multinational, before-and-after intervention trial. Crit Care Med. 2020;48(2):176-184. doi:10.1097/CCM.0000000000004113

41. Angus DC, Truog RD. Toward better ICU use at the end of life. JAMA. 2016;315(3):255-256. doi:10.1001/jama.2015.18681

42. Sprung CL, Ricou B, Hartog CS, et al. Changes in end-of-life practices in European intensive care units from 1999 to 2016. JAMA. 2019;322(17):1692-1704. doi:10.1001/jama.2019.14608

43. Ma J, Chi S, Buettner B, et al. Early palliative care consultation in the medical ICU: a cluster randomized crossover trial. Crit Care Med. 2019;47(12):1707-1715. doi:10.1097/CCM.0000000000004016

44. Lobo SM, De Simoni FHB, Jakob SM, et al. Decision-making on withholding or withdrawing life support in the ICU: a worldwide perspective. Chest. 2017;152(2):321-329. doi:10.1016/j.chest.2017.04.176

45. Benoit DD, Jensen HI, Malmgren J, et al. Outcome in patients perceived as receiving excessive care across different ethical climates: a prospective study in 68 intensive care units in Europe and the USA. Intensive Care Med. 2018;44(7):1039-1049. doi:10.1007/ s00134-018-5231-8

46. Flannery L, Ramjan LM, Peters K. End-of-life decisions in the Intensive Care Unit (ICU) - exploring the experiences of ICU nurses and doctors - a critical literature review. Aust Crit Care. 2016;29 (2):97-103. doi:10.1016/j.aucc.2015.07.004

47. Pereira SM, Teixeira CM, Carvalho AS, Hernández-Marrero $P$ Compared to palliative care, working in intensive care more than doubles the chances of burnout: results from a nationwide comparative study. PLoS One. 2016;11(9):1-21. doi:10.1371/journal. pone. 0162340

48. Nordenskjöld Syrous A, Ågård A, Kock Redfors M, Naredi S, Block L. Swedish intensivists' experiences and attitudes regarding end-of-life decisions. Acta Anaesthesiol Scand. 2020;64(5):656-662. doi:10.1111/aas.13549

49. Gerritsen RT, Hartog CS, Curtis JR. New developments in the provision of family-centered care in the intensive care unit. Intensive Care Med. 2017;43(4):550-553. doi:10.1007/s00134-017-4684-5

50. White DB, Angus DC, Shields A-M, et al. A randomized trial of a family-support intervention in intensive care units. $N$ Engl $J$ Med. 2018;378(25):2365-2375. doi:10.1056/nejmoa1802637

51. Scheunemann LP, Ernecoff NC, Buddadhumaruk P, et al. Clinicianfamily communication about patients' values and preferences in intensive care units. JAMA Intern Med. 2019;179(5):676-684. doi:10.1001/jamainternmed.2019.0027

52. White DB, Carson S, Anderson W, et al. A multicenter study of the causes and consequences of optimistic expectations about prognosis by surrogate decision-makers in ICUs. Crit Care Med. 2019;47 (9):1184-1193. doi:10.1097/CCM.0000000000003807

53. Wilson ME, Dobler CC, Zubek L, et al. Prevalence of disagreement about appropriateness of treatment between ICU patients/surrogates and clinicians. Chest. 2019;155(6):1140-1147. doi:10.1016/j. chest.2019.02.404

54. Barwise AK, Nyquist CA, Espinoza Suarez NR, et al. End-of-life decision-making for ICU patients with limited English proficiency: a qualitative study of healthcare team insights. Crit Care Med. 2019;47(10):1380-1387. doi:10.1097/CCM.0000000000003920
55. Barwise A, Jaramillo C, Novotny P, et al. Differences in code status and end-of-life decision making in patients with limited English proficiency in the intensive care unit. Mayo Clin Proc. 2018;93 (9):1271-1281. doi:10.1016/j.mayocp.2018.04.021

56. Sviri S, Geva D, vanHeerden PV, et al. Implementation of a structured communication tool improves family satisfaction and expectations in the intensive care unit. J Crit Care. 2019;51:6-12. doi:10.1016/j.jcrc.2019.01.011

57. Kentish-Barnes N, Seegers V, Legriel S, et al. CAESAR: a new tool to assess relatives' experience of dying and death in the ICU. Intensive Care Med. 2016;42(6):995-1002. doi:10.1007/s00134-016-4260-4

58. Lee J, Abrukin L, Flores S, et al. Early intervention of palliative care in the emergency department during the COVID-19 pandemic. JAMA Intern Med. 2020;180(9):1252-1254. doi:10.1001/jamainternmed. 2020.2713

59. Kerkham T, Brain M. Goals of care conversations and documentation in patients triggering medical emergency team calls. Intern Med J. 2020;50(11):1373-1376. doi:10.1111/imj.14667

60. George N, Phillips E, Zaurova M, Song C, Lamba S, Grudzen C. Palliative care screening and assessment in the emergency department: a systematic review. J Pain Symptom Manage. 2016;51 (1):108-119.e2. doi:10.1016/j.jpainsymman.2015.07.017

61. Lafond P, Chalayer E, Roussier M, Weber E, Lacoin-Reynaud Q, Tardy B. A hospice and palliative care bed dedicated to patients admitted to the emergency department for end-of-life care. Am J Hosp Palliat Med. 2016;33(4):403-406. doi:10.1177/1049909114562947

62. Crooms RC, Gelfman LP. Palliative care and end-of-life considerations for the frail patient. Anesth Analg. 2020;130(6):1504-1515. doi:10.1213/ANE.0000000000004763

63. Candido KD, Kusper TM, Knezevic NN. New cancer pain treatment options. Curr Pain Headache Rep. 2017;21(2):12. doi:10.1007/ s11916-017-0613-0

64. Fine PG, Davis MS, Muir JC, Byas-Smith M. Anesthesiology and palliative care: past, present, and future. Anesth Analg. 2018;127 (1):12-14. doi:10.1213/ANE.0000000000002870

65. Barello S, Palamenghi L, Graffigna G. Burnout and somatic symptoms among frontline healthcare professionals at the peak of the Italian COVID-19 pandemic. Psychiatry Res. 2020;290:113129. doi:10.1016/j.psychres.2020.113129

66. Huang Y, Zhao N. Generalized anxiety disorder, depressive symptoms and sleep quality during COVID-19 outbreak in China: a web-based cross-sectional survey. Psychiatry Res. 2020;288:112954. doi:10.1016/j.psychres.2020.112954

67. Chew NWS, Lee GKH, Tan BYQ, et al. A multinational, multicentre study on the psychological outcomes and associated physical symptoms amongst healthcare workers during COVID-19 outbreak. Brain Behav Immun. 2020;88:559-565. doi:10.1016/j. bbi.2020.04.049

68. Robert R, Kentish-Barnes N, Boyer A, Laurent A, Azoulay E, Reignier J. Ethical dilemmas due to the Covid-19 pandemic. Ann Intensive Care. 2020;10(1):1-9. doi:10.1186/s13613-020-00702-7

69. World Health Organization. Rational Use of Personal Protective Equipment for COVID-19 and Considerations During Severe Shortages. World Health Organization; 2020.

70. Bloomer MJ, Bouchoucha S. Australian College of Critical Care Nurses and Australasian College for Infection Prevention and Control position statement on facilitating next-of-kin presence for patients dying from coronavirus disease 2019 (COVID-19) in the intensive care unit. Aust Crit Care. 2021;34(2):132-134. doi:10.1016/j.aucc.2020.07.002

71. Vergano M, Bertolini G, Giannini A, et al. Siaarti recommendations for the allocation of intensive care treatments in exceptional, resource-limited circumstances. Minerva Anestesiol. 2020;86 (5):469-472. doi:10.23736/S0375-9393.20.14619-4 
72. Zandifar A, Badrfam R, Yazdani S, et al. Prevalence and severity of depression, anxiety, stress and perceived stress in hospitalized patients with COVID-19. J Diabetes Metab Disord. 2020;19 (2):1431-1438. doi:10.1007/s40200-020-00667-1

73. Montauk TR, Kuhl EA. COVID-related family separation and trauma in the intensive care unit. Psychol Trauma Theory Res Pract Policy. 2020;12(S1):96-97. doi:10.1037/tra0000839

74. Azoulay E, Kentish-Barnes N. A 5-point strategy for improved connection with relatives of critically ill patients with COVID-19. Lancet Respir Med. 2020;8(6):e52. doi:10.1016/S2213-2600(20)30223-X

75. Kentish-Barnes N, Morin L, Cohen-Solal Z, Cariou A, Demoule A, Azoulay E. The lived experience of ICU clinicians during the Coronavirus disease 2019 outbreak: a qualitative study. Crit Care Med. 2021;49(6):e585-e597. doi:10.1097/ccm.0000000000004939

76. Piscitello GM, Fukushima CM, Saulitis AK, et al. Family meetings in the intensive care unit during the Coronavirus disease 2019 pandemic. Am J Hosp Palliat Med. 2021;38(3):305-312. doi:10.1177/1049909120973431

77. Curtis JR, Kross EK, Stapleton RD. The importance of addressing advance care planning and decisions about do-not-resuscitate orders during Novel Coronavirus 2019 (COVID-19). JAMA. 2020;323 (18):1771-1772. doi:10.1001/jama.2020.4894

78. Ippolito M, Catalisano G, Marino C, et al. Mortality after in-hospital cardiac arrest in patients with COVID-19: a systematic review and meta-analysis. Resuscitation. 2021;164:122-129. doi:10.1016/j. resuscitation.2021.04.025

79. Obata R, Maeda T, Rizk D, Kuno T. Palliative care team involvement in patients with COVID-19 in New York City. Am J Hosp Palliat Med. 2020;37(10):869-872. doi:10.1177/1049909120940986

80. Schoenherr LA, Cook A, Peck S, Humphreys J, Goto Y. Since January 2020 Elsevier has created a COVID-19 resource centre with free information in English and Mandarin on the novel coronavirus COVID- 19. The COVID-19 resource centre is hosted on Elsevier Connect, the company' $s$ public news and information. 2020 January.

81. Moriyama D, Scherer JS, Sullivan R, Lowy J, Berger JT. The impact of COVID-19 surge on clinical palliative care: a descriptive study from a New York hospital system. J Pain Symptom Manage. 2021;61 (3):e1-e5. doi:10.1016/j.jpainsymman.2020.12.011

82. Sheehan J, Ho KS, Poon J, Sarosky K, Fung JY. Palliative care in critically ill COVID-19 patients: the early New York City experience. BMJ Support Palliat Care. 2020;(9):1-5. doi:10.1136/bmjspcare2020-002677
83. Mottiar M, Hendin A, Fischer L, Roze Des Ordons A, Hartwick M. End-of-life care in patients with a highly transmissible respiratory virus: implications for COVID-19. Can J Anesth. 2020;67 (10):1417-1423. doi:10.1007/s12630-020-01699-0

84. Andrews LJ, Benken ST. COVID-19: ICU delirium management during SARS-CoV-2 pandemic - pharmacological considerations. Crit Care. 2020;24(1):1-9. doi:10.1186/s13054-020-03072-5

85. Fausto J, Hirano L, Lam D, et al. Creating a palliative care inpatient response plan for COVID-19- the UW medicine experience. J Pain Symptom Manage. 2020;60(1):e21-e26. doi:10.1016/j. jpainsymman.2020.03.025

86. Karthik A, Rustagi K, Mishra S, et al. Effect of nation-wide lockdown on palliative care services in a tertiary care centre in India: a retrospective observational study. Indian J Palliat Care. 2020;26 (5):S45-S47. doi:10.4103/IJPC.IJPC 14220

87. Mehta AK, Smith TJ. Palliative care for patients with cancer in the COVID-19 era. JAMA Oncol. 2020;6(10):1527-1528. doi:10.1001/ jamaoncol.2020.1938

88. Khurana D, Raheja S, Dayal M, Di P, Ganapathy U. COVID 19: the new normal in the clinic: overcoming challenges in palliative care. Indian $J$ Palliat Care. 2020;26(5):S81-S85. doi:10.4103/IJPC.IJPC_173_20

89. Anesi GL, Liu VX, Gabler NB, et al. Associations of intensive care unit capacity strain with disposition and outcomes of patients with sepsis presenting to the Emergency Department. Ann Am Thorac Soc. 2018;15(11):1328-1335. doi:10.1513/AnnalsATS.201804-241OC

90. Rubio O, Estella A, Cabre L, et al. Ethical recommendations for a difficult decision-making in intensive care units due to the exceptional situation of crisis by the COVID-19 pandemia: a rapid review \& consensus of experts. Med Intensiva. 2020;44(7):439-445. doi:10.1016/j.medin.2020.04.006

91. Krishna LKR, Neo HY, Chia EWY, et al. The role of palliative medicine in ICU bed allocation in COVID-19: a joint position statement of the Singapore Hospice Council and the Chapter of Palliative Medicine Physicians. Asian Bioeth Rev. 2020;12(2):205-211. doi:10.1007/s41649-020-00128-0

92. Page MJ, McKenzie JE, Bossuyt PM, Boutron I, Hoffmann TC, Mulrow CD, et al. The PRISMA 2020 statement: an updated guideline for reporting systematic reviews. BMJ. 2021;372:n71. doi:10.1136/bmj.n7
Journal of Multidisciplinary Healthcare

\section{Publish your work in this journal}

The Journal of Multidisciplinary Healthcare is an international, peerreviewed open-access journal that aims to represent and publish research in healthcare areas delivered by practitioners of different disciplines. This includes studies and reviews conducted by multidisciplinary teams as well as research which evaluates the results or conduct of such teams or healthcare processes in general. The journal

\section{Dovepress}

covers a very wide range of areas and welcomes submissions from practitioners at all levels, from all over the world. The manuscript management system is completely online and includes a very quick and fair peer-review system. Visit http://www.dovepress.com/testimonials. php to read real quotes from published authors. 